\title{
ALGEBRAIC RELATIONS AMONG SOLUTIONS OF LINEAR DIFFERENTIAL EQUATIONS
}

\author{
MICHAEL F. SINGER
}

\begin{abstract}
Using power series methods, Harris and Sibuya $[3,4]$ recently showed that if $k$ is an ordinary differential field of characteristic zero and $y \neq 0$ is an element of a differential extension of $k$ such that $y$ and $1 / y$ satisfy linear differential equations with coefficients in $k$, then $y^{\prime} / y$ is algebraic over $k$. Using differential galois theory, we generalize this and characterize those polynomial relations among solutions of linear differential equations that force these solutions to have algebraic logarithmic derivatives. We also show that if $f$ is an algebraic function of genus $\geq 1$ and if $y$ and $f(y)$ or $y$ and $e^{\int y}$ satisfy linear differential equations, then $y$ is an algebraic function.
\end{abstract}

1. Introduction. In $[3,4]$, Harris and Sibuya proved the followng:

PROPOSITION 1. Let $k$ be an ordinary differential field of characteristic 0 and let $L_{1}(Y)$ and $L_{2}(Y)$ be nonzero homogeneous linear differential polynomials with coefficients in $k$. Let $K$ be a differential extension of $k$ and $y_{1}$ and $y_{2}$ nonzero elements of $K$ such that $L_{1}\left(y_{1}\right)=L_{2}\left(y_{2}\right)=0$.

(a) If $y_{1} y_{2}=1$, then $y_{1}^{\prime} / y_{1}=-y_{2}^{\prime} / y_{2}$ is algebraic over $k$.

(b) If $y_{1}=y_{2}^{m}$ for some positive integer $m$ such that the order of $L_{1} \leq m$, then $y_{1}^{\prime} / y_{1}=m y_{2}^{\prime} / y_{2}$ is algebraic over $k$.

In [12], Sperber, using some elementary commutative algebra, gave these results a uniform treatment that allowed for the following generalization:

PROPOSITION 2. Let $k$ be an ordinary differential field of characteristic 0 and let $L_{i}(Y), i=1, \ldots, n$, be nonzero homogeneous linear differential polynomials with coefficients in $k$. Let $K$ be a differential extension of $k$ and $y_{i}, i=1, \ldots, n$, nonzero elements of $K$ such that $L_{i}\left(y_{i}\right)=0$ for $i=1, \ldots, n$. If $y_{1}=y_{2}^{m_{2}} \cdots y_{n}^{m_{n}}$, for positive integers $m_{2}, \ldots, m_{n}$ and the order of $L_{1} \leq \min \left\{m_{2}, \ldots, m_{n}\right\}$, then $y_{i}^{\prime} / y_{i}$ is algebraic over $k$ for each $i=1, \ldots, n$.

Proposition 1(a) is obtained by letting $n=3, m_{2}=m_{3}=1$ and $y_{1}=1$. Proposition 1(b) is obtained by letting $n=2$ and $m_{2}=m$. Sperber's techniques also allow him to handle solutions of certain nonlinear diferential equations.

In this paper we prove results that imply

PROPOSITION 3. Let $k \subset K$ be ordinary differential fields of characteristic 0 with the constants of $K\left(\right.$ i.e., the set of $c \in K$ such that $\left.c^{\prime}=0\right)$ algebraic over $k$. For $i=1,2,3$, let $y_{i}$ be a nonzero element of $K$ and $L_{i}(Y)$ be a nonzero homogeneous

Received by the editors June 18,1985 .

1980 Mathematics Subject Classification. Primary $12 \mathrm{HO}$.

Key words and phrases. Linear differential equations, Picard-Vessiot theory, linear algebraic groups. 
linear differential polynomial with coefficients in $k$ such that $L_{i}\left(y_{i}\right)=0$. Assume $y_{1}=y_{2} y_{3}^{m}$ for some positive integer $m$. if the order of $L_{1}(Y) \leq m$, then $y_{3}^{\prime} / y_{3}$ is algebraic over $k$.

We can deduce Proposition 2 from Proposition 3 only under the additional assumption that the constants of $K$ are algebraic over $k$. This annoying assumption is forced on us by our techniques (differential galois theory) but does not interfere with the applications that the authors of $[3,4$ and 12] had in mind. To deduce Proposition 2 with this added assumption, let $y_{1}=\left(y_{2}^{m_{2}} \ldots y_{n-1}^{m_{n-1}}\right) y_{n}^{m_{n}}$. Since each $y_{i}$ satisfies a linear differential equation over $k, y_{2}^{m_{2}} \cdots y_{n-1}^{m_{n-1}}$ will also satisfy a linear differential equation over $k$. By assumption, $y_{1}$ satisfies a homogeneous linear differential equation, over $k$, of order $\leq \min \left\{m_{2}, \ldots, m_{n}\right\} \leq m_{n}$. Proposition $3 \mathrm{im}$ plies that $y_{r}^{\prime} / y_{r}$ is algebraic over $k$. Regrouping, the other $y_{i}$ are handled similarly. Note that if we remove the assumption that the order of $L_{1}(Y) \leq \min \left\{m_{2}, \ldots, m_{n}\right\}$ in Proposition 2, Proposition 3 allows us to still conclude that $y_{i}^{\prime} / y_{i}$ is algebraic over $k$ for each $i \geq 2$ such that the order of $L_{1} \leq m_{i}$.

Proposition 3 is a consequence of a more general result proved in $\S 3$. In that section we also prove results that imply the results mentioned in the abstract. In $\S 2$ we prove a group theoretic result that is the technical heart of this paper. We wish to thank E. Kolchin, W. Lichtenstein, A. Magid and S. Sperber for many helpful comments and W. Harris, Y. Sibuya and S. Sperber for giving us preprints of their papers.

2. Group theory. Our main result in this section is a generalization of the following theorem of Rosenlicht $[\mathbf{1 0}, \mathbf{7}]$ : Let $G$ be a connected linear algebraic group and $y_{1}, y_{2}$ regular functions on $G$ such that $y_{1} y_{2}=1$. Then $y_{1}$ and $y_{2}$ are constant multiples of characters. Theorem 1, below, characterizes those polynomials $P\left(Y_{1}, \ldots, Y_{n}\right)$ such that if $y_{1}, \ldots, y_{n}$ are regular functions on a connected linear algebraic group and $P\left(y_{1}, \ldots, y_{n}\right)=0$, then each $y_{i}, i=1, \ldots, n$, must be a constant multiple of a character (e.g., $\left.P\left(Y_{1}, Y_{2}\right)=Y_{1} Y_{2}-1\right)$.

Let $k$ be an algebraically closed field of characteristic zero and $G$ a connected linear algebraic group defined over $k$. We denote by $k(G)$ (resp. $k[G]$ ) the field of rational functions on $G$ (resp. the ring of regular functions on $G$ ). If $g$ is a $k$-point of $G$, we denote by $\rho_{g}$ (resp. $\lambda_{g}$ ) the regular map defined by $\rho_{g}(h)=h g$ for $h$ in $G$ (resp. $\left.\lambda_{g}(h)=g^{-1} h\right) . \rho_{g}$ and $\lambda_{g}$ induce automorphisms of $k(G)$ which we denote by $\rho_{g}^{*}$ and $\lambda_{g}^{*}$. Note that $\rho_{g}^{*}$ and $\lambda_{g}^{*}$ restrict to automorphisms of $k[G]$. For $y \in k(G)$, we let $k y^{G}$ denote the $k$-span of $\left\{\rho_{g}^{*} y \mid g\right.$ a $k$-point of $\left.G\right\}$. It is well known [1, p. 106] that for $y \in k(G), k y^{G}$ has finite dimension if and only if $y \in k[G]$.

Let $\left(N_{1}, \ldots, N_{n}\right)$ be an $n$-tuple of positive integers and $S$ a subset of $\{1, \ldots, n\}$. Let $I$ be a set of polynomials in $k\left[Y_{1}, \ldots, Y_{n}\right]$. We say that $I$ has property (A) (resp. $\left.\left(\mathrm{A}^{\prime}\right)\right)$ for $\left(N_{1}, \ldots, N_{n}\right)$ with respect to $S$ if:

For any connected linear $k$-group $G$ and any $y_{1}, \ldots, y_{n}$ in $k(G)-$ $\{0\}$ such that the dimension of $k y_{i}^{G} \leq N_{i}$ for $i=1, \ldots, n$, if $P\left(y_{1}, \ldots, y_{n}\right)=0$ for all $P$ in $I$, then for each $i$ in $S, y_{i}$ is a $k$-multiple of a character of $G$ (resp. $y_{i}$ is in $k$ ).

For example, Rosenlicht's theorem implies that for $r=2, N_{1}$ and $N_{2}$ arbitrary and $S=\{1,2\}$, the singleton $\left\{Y_{1} Y_{2}-1\right\}$ has property (A) for $\left(N_{1}, N_{2}\right)$ with respect 
to $S$. Theorem 1 below implies that $\left\{Y_{1}-Y_{2} Y_{3}^{m}\right\}$ has property (A) for $\left(m, N_{2}, N_{3}\right)$ with respect to $\{3\}$, where $m, N_{2}$ and $N_{3}$ are arbitrary positive integers. Theorem 1 also shows that if the zero set of $I$ in $k^{n}$ is a curve of genus $\geq 1$, then, for $N_{1}, \ldots, N_{n}$ arbitrary positive integers and $S=\{1, \ldots, n\}, I$ has property $\left(\mathrm{A}^{\prime}\right)$ for $\left(N_{1}, \ldots, N_{n}\right)$ with respect to $S$.

We wish to give algebraic criteria for properties $(\mathrm{A})$ and $\left(\mathrm{A}^{\prime}\right)$. Theorem 1 says that the following are such criteria: Let $\left(N_{1}, \ldots, N_{n}\right), S$ and $I$ be as shown. We say that $I$ has property (B) (resp. $\left.\left(\mathrm{B}^{\prime}\right)\right)$ for $\left(N_{1}, \ldots, N_{n}\right)$ with respect to $S$ if:

For any $u_{1}, \ldots, u_{n}$ in $k\left[t, t^{-1}\right](t$ transcendental over $k)$ with each $u_{i}$ having at most $N_{i}$ nonzero terms, if $P\left(u_{1}, \ldots, u_{n}\right)=0$, then for each $i$ in $S, u_{i}$ is a monomial in $k\left[t, t^{-1}\right]$ (resp. $u_{i}$ is in $k$ ).

For example, let $n=2, S=\{1,2\}$, and $N_{1}$ and $N_{2}$ arbitrary positive integers. The singleton $I=\left\{Y_{1} Y_{2}-1\right\}$ has property (B) for $\left(N_{1}, N_{2}\right)$ with respect to $\{1,2\}$. This follows from the fact that the only invertible elements of $k\left[t, t^{-1}\right]$ are monomials. If we let $n, N_{1}, \ldots, N_{n}$ be arbitrary positive integers and $S=\{1, \ldots, n\}$ and let $I$ be a set of polynomials in $k\left\{Y_{1}, \ldots, Y_{n}\right\}$ such that the zero set of $I$ in $k^{n}$ is a curve of genus $\geq 1$, then $I$ has property $\left(\mathrm{B}^{\prime}\right)$ for $\left(N_{1}, \ldots, N_{n}\right)$ with respect to $S$. This follows from the stronger fact that if $u_{1}, \ldots, u_{n}$ are in $k(t)$ and $P\left(u_{1}, \ldots, u_{n}\right)=0$ for all $P$ in $I$, then $u_{1}, \ldots, u_{n}$ are in $k$.

A less trivial example is given by the following. Let $n=3$ and let $m$ be a positive integer. Let $I=\left\{Y_{1}-Y_{2} Y_{3}^{m}\right\}$. We claim that $I$ has property (B) for $\left(m, N_{2}, N_{3}\right)$ with respect to $\{3\}$, where $N_{2}$ and $N_{3}$ are arbitrary positive integers. To see this, we must show that if $u_{1}, u_{2}, u_{3}$ are elements of $k\left[t, t^{-1}\right]$ such that $u_{1}-u_{2} u_{3}^{m}=0$ and $u_{1}$ has at most $m$ nonzero terms, then $u_{3}$ is a monomial. It suffices to show, given $u_{2}, u_{3}$ in $k\left[t, t^{-1}\right]$ with $u_{3}$ having more than one nonzero term, that $u_{2} u_{3}^{m}$ has more than $m$ nonzero terms. We may assume that $u_{2}$ and $u_{3}$ are in $k[t]$. If $u_{3}$ has more than one nonzero term, then $u_{3}=0$ has a nonzero root. In this case $u_{2} u_{3}^{m}$ has a nonzero root of multiplicity at least $m$. Therefore, it is enough to show that for $v \in k[t]$, if $v$ has a nonzero root of multiplicity $\geq n$ then $v$ has more than $n$ nonzero terms. This will be proved by induction on the degree of $v$. If the degree of $v$ is one, the conclusion is obvious. If the degree of $v$ is bigger than 1 , we may assume $v(0) \neq 0$. Applying the induction hypothesis to $d v / d t$ and noting that $v$ has one more nonzero term than $d v / d t$, we reach the desired conclusion.

The main result of this section is

THEOREM 1. Let $k,\left(N_{1}, \ldots, N_{n}\right), S$ and $I$ be as above, I has property (A) (resp. $\left.\left(\mathrm{A}^{\prime}\right)\right)$ for $\left(N_{1}, \ldots, N_{n}\right)$ with respect to $S$ if and only if $I$ has property (B) (resp. $\left.\left(\mathrm{B}^{\prime}\right)\right)$ for $\left(N_{1}, \ldots, N_{n}\right)$ with respect to $S$.

To prove this, we need the following elementary lemmas. Lemma 1 shows that our property (B) implies Sperber's property of being $\left(N_{1}-1, \ldots, N_{n}-1\right)$-polynomial free $[12$, p. 7$]$.

LEMMA 1. Let $k,\left(N_{1}, \ldots, N_{r}\right), S$ and $I$ be as above. If $I$ has property (B) for $\left(N_{1}, \ldots, N_{r}\right)$ with respect to $S$, then $I$ has the following property: if $u_{1}, \ldots, u_{n}$ are elements of $k[t],\left(t\right.$ transcendental over $k$ ) with the degree of $u_{i} \leq N_{i}-1$ for $i=1 \ldots, n$ and $P\left(u_{1}, \ldots, u_{n}\right)=0$ for all $P$ in $I$, then for each $i$ in $S, u_{i} \in k$. 
PROOF. Let $u_{1}, \ldots, u_{n}$ be in $k[t]$ with the degree of each $u_{i} \leq N_{i}-1$ for $i=1, \ldots, r$ and $P\left(u_{1}, \ldots, u_{n}\right)=0$ for all $P$ in $I$. Since $I$ has property (B) and $k[t] \subset k\left[t, t^{-1}\right]$, we have for each $i$ in $S, u_{i}=a_{i} t^{m_{i}}$ with $a_{i} \in k$ and $m_{i}$ a nonegative integer. Replacing $t$ by $t+1$, we have $P\left(a_{1}(t+1)^{m_{1}}, \ldots, a_{n}(t+1)^{m_{n}}\right)=0$ for all $P$ in $I$. By property (B), we have for each $i \in S, a_{i}(t+1)^{m_{i}}$ must be a monomial, so $m_{i}=0$ and $u_{i}=a_{i} \in k$.

In the following two lemmas, $G_{a}$ will denote the additive group $k$ and $G_{m}$ the multiplicative group $k-\{0\}$. Note that if $t$ is transcendental over $k$, we may identify $k\left[G_{a}\right]$ with $k[t]$ and $k\left[G_{m}\right]$ with $k\left[, t, t^{-1}\right]$.

LEMMA 2. Let $k$ be an algebraically closed field of characteristic 0 and $N$ a positive integer. If $u \in k\left[G_{a}\right]=k[t]$, then the dimension of $k u^{G_{a}}$ is at most $N$ if and only if the degree of $u$, as a polynomial in $t$, is at most $N-1$.

ProOF. Note that for $c \in k=G_{a}$ and $u(t) \in k\left[G_{a}\right]=k[t], \rho_{c}^{*}(u)=u(t+c)$. This implies that the vector space of all polynomials of degree $\leq N-1$ is left stable by $\rho_{c}^{*}$ for $c \in G_{a}$. Therefore if $u \in k\left[G_{a}\right]$ is a polynomial of degree at most $N-1$, the dimension of $k y^{G_{a}} \leq N$.

Conversely, assume that the dimension of $k u^{G_{a}} \leq N$. We define a derivation $D$ on $k(t)$ by letting $D t=1$ and $D c=0$ for all $c \in k$. Note that for any $c \in k, \rho_{c}^{*}$ commutes with $D$. Let $u_{1}, \ldots, u_{m}$ be a basis for $k u^{G_{a}}$ and let

$$
L(y)=\operatorname{Wr}\left(y, u_{1}, \ldots, u_{m}\right) / \operatorname{Wr}\left(u_{1}, \ldots, u_{m}\right),
$$

where $\mathrm{Wr}$ denotes the Wronskian determinant. The coefficients of $L(y)$ are left fixed by $\rho_{c}^{*}$ for $c \in k$, so these coefficients lie in $k$. Let $L(y)=y^{(m)}+a_{m-1} y^{(m-1)}+$ $\cdots+a_{i} y^{(i)}$, with $a_{i} \neq 0$. For $v \in k[t]$, if $L(v)=0$ then the degree of $v$ is at most $i-1 \leq m-1 \leq N-1$. Since $L(v)=0$ for all $v \in k u^{G_{a}}$, we have that the degree of $u$ is at most $N-1$.

LEMMA 3. Let $k$ be an algebraically closed field of characteristic 0 and $N$ a positive integer. If $u \in k\left[G_{m}\right]=k\left[t, t^{-1}\right]$, then the dimension of $k u^{G_{m}}$ is at most $N$ if and only if $u$ contains at most $N$ nonzero terms.

PROOF. Note that for $c \in k-\{0\}=G_{m}$ and $u(t) \in k\left[G_{m}\right]=k\left[t, t^{-1}\right], \rho_{c}^{*}(u)=$ $u(t c)$. A similar argument to that appearing in the proof of Lemma 2 shows that if $u$ has at most $N$ nonzero terms, then the dimension of $k u^{G_{m}}$ is at most $N$.

Conversely, assume that the dimension of $k u^{G_{m}}$ is at most $N$. Define a derivation $D$ on $k(t)$ by letting $D t=t$ and $D c=0$ for all $c$ in $k$. D commutes with $\rho_{c}^{*}$ for all $c \in k-\{0\}$. Let $u_{1}, \ldots, u_{m}$ be the basis of $k u^{G_{m}}$ and let $L(y)$ be defined as in the proof of Lemma 2. We again see that $L(y)$ has coefficients in $k$. If $L(y)=y^{(m)}+a_{m-1} y^{(m-1)}+\cdots+a_{0} y$, let $p(y)=y^{m}+a_{m-1} y^{m-1}+\cdots+a_{0}$. If $u=a_{1} t^{m_{1}}+\cdots+a_{s} t^{m_{s}}$, where the $m_{i}$ are distinct integers and the $a_{i}$ are nonzero elements of $k$, then $L(u)=p\left(m_{1}\right) a_{1} t^{m_{1}}+\cdots+p\left(m_{s}\right) a_{s} t^{m_{s}}$. Since $L(u)=0$, we have that $p\left(m_{i}\right)=0$ for $i=1, \ldots, r$. Therefore $r \leq m \leq N$.

We remark that we could replace the argument involving $D$ in Lemma 3 with an argument involving matrices that resemble Vandermonde matrices, yielding a proof true in all characteristics. Lemma 2, on the other hand, is true only in characteristic 0 , since if $k$ is a field of characteristic $p \neq 0$ and $u$ is a $p$-polynomial (i.e., a linear combination of terms $\left.t^{p^{i}}, i \geq 0\right)$ then $k u^{G_{m}}$ will have dimension at most 2 . 
PROOF OF THEOREM 1. Let us first assume that $I$ has property (B) for $\left(N_{1}, \ldots, N_{n}\right)$ with respect to $S$. Let $G$ be a connected linear $k$-group, and let $y_{1}, \ldots, y_{n}$ be elements of $k(G)=\{0\}$ such that the dimension of $k y_{i}^{G} \leq N_{i}$ for $i=1, \ldots, n$. As we have noted, this implies that each $y_{i} \in k[G]$. We also note that we may assume that $y_{i}(e) \neq 0$ for $i=1, \ldots, n$, where $e$ is the identity of $G$. To see this, let $g \in G$ satisfy $\left(y_{1} \cdots y_{n}\right)(g) \neq 0$. We then have $\left(\rho_{g}^{*}\left(y_{1}\right) \cdots \rho_{g}^{*}\left(y_{n}\right)\right)(e) \neq 0$. $\rho_{g}^{*}\left(y_{i}\right)$ is a $k$-multiple of a character if and only if $y_{i}$ is and the hypotheses regarding $y_{i}$ apply as well to $\rho_{g}^{*}\left(y_{i}\right)$. Therefore, we may replace the $y_{i}$ by $\rho_{g}^{*}\left(y_{i}\right)$ if need be. In particular, we may asusme that for each $i=1, \ldots, n, y_{i}$, when restricted to any subgroup of $G$, is not identically zero.

We shall first show that for any unipotent $h$ in $G, \rho_{h}^{*}\left(y_{i}\right)=y_{i}$ for all $i$ in $S$. Let $0=\left\{g \in G \mid y_{i}(g) \neq 0\right.$ for $\left.i=1, \ldots, n\right\}$. Since $O$ is Zariski dense in $G$, it suffices to show that for $g$ in $O$ and $i$ in $S, y_{i}(g)=y_{i}(g h)$. Let $H$ be a subgroup of $G$, isomorphic to $G_{a}$, containing $h\left[5\right.$, p. 96] and let $u_{i}$ be the restriction of $\lambda_{g^{-1}}^{*}\left(y_{i}\right)$ to $H$. Note that $u_{i} \not \equiv 0$ for $i=1, \ldots, n$. We may identify $u_{i}$ with an element in $k[H]=k[t]$. We furthermore have that the dimension of $k u_{i}^{H} \leq N_{i}$ for $i=1, \ldots, n$, so by Lemma $2, u_{i}$ is of degree at most $N_{i}-1$. Lemma 1 implies that for each $i$ in $S, u_{i}$ is in $k$. Therefore $y_{i}(g)=u_{i}(e)=u_{i}(h)=y_{i}(g h)$.

We now note that to finish the proof, it suffices to show that for any torus $T \subset G$, and any $i$ in $S$, the restriction of $y_{i}$ to $T$ is a nonzero $k$-multiple of a character of $T$ and therefore never zero on $T$. Assuming this to be true, let $g \in G$ and let $g=g_{s} g_{u}$,

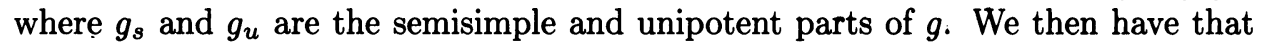
$y_{i}(g)=y_{i}\left(g_{s} g_{u}\right)=y_{i}\left(g_{s}\right)$. Since $g_{s}$ belongs to some torus [5, p. 124 and p. 139], we have $y_{i}\left(g_{s}\right) \neq 0$. Therefore $y_{i}$ is never zero on $G$ and so is invertible in $k[G]$. By Rosenlicht's theorem, we have that $y_{i}$ is a $k$-multiple of a character.

Let $T=G_{m} \times \cdots \times G_{m}$ be a torus and let $\overline{y_{i}}, i=1, \ldots, n$, be the restriction of $y_{i}$ to $T$. We may identify $k[T]$ with $k\left[X_{1}, \ldots, X_{s},\left(X_{1}, \ldots ; X_{s}\right)^{-1}\right]$ for some $s$, and we shall identify the $\overline{y_{i}}$ with elements of this latter ring. We wish to show that for any $i$ in $S, \overline{y_{i}}$ is a nonzero monomial in $X_{1}, \ldots, X_{s}$. If some $\overline{y_{i}}$, with $i$ in $S$, contains more than one nonzero term, then one of the $X_{i}$, say $X_{1}$, appears with different exponents in at least two terms. Write $\overline{y_{i}}=\sum a_{i j} X_{1}^{j}$, where the $a_{i j}$ are in $k\left[X_{2}, \ldots, X_{s},\left(X_{2} \cdots X_{s}\right)^{-1}\right]$. Let $c_{2}, \ldots, c_{s}$ be nonzero elements of $k$ such that $a_{i j}\left(c_{2}, \ldots, c_{s}\right) \neq 0$ for all $i$ and $j$ with $a_{i j} \neq 0$, and let $u_{i}=\overline{y_{i}}\left(t, c_{2}, \ldots, c_{s}\right) \in$ $k\left[t, t^{-1}\right]$. Let $H$ be the first copy of $G_{m}$ in $T=G_{m} \times \cdots \times G_{m}$ and identify $k\left[t, t^{-1}\right]$ with $k[H]$. By our hypotheses and Lemma 3 , we have that, for each $i$ in $S, u_{i}$ is a monomial in $k\left[t, t^{-1}\right]$, violating our constructions of the $u_{i}$. Therefore, for each $i$ in $S, \overline{y_{i}}$ is a monomial, and the restriction of $y_{i}$ to any torus is a $k$-multiple of a character.

Now assume that $I$ has property $\left(\mathrm{B}^{\prime}\right)$ for $\left(N_{1}, \ldots, N_{n}\right)$ with respect to $S$ and let $G$ and $y_{1}, \ldots, y_{n}$ be as above. We wish to show that for $i$ in $S, y_{i}$ is in $k$. To do this, it is enough to show that if $g$ is unipotent or semisimple, then $\rho_{g}^{*}\left(y_{i}\right)=y_{i}$. Since property $\left(\mathrm{B}^{\prime}\right)$ implies property $(\mathrm{B})$, the first part of the above proof shows that $\rho_{g}^{*}\left(y_{i}\right)=y_{i}$ for unipotent $g$. Since any semisimple element lies in a torus and every torus is isomorphic to a product of copies of $G_{m}$, it suffices to show that, if $H$ is a copy of $G_{m}$ in $G$, then, for $i$ in $S, y_{i}$ restricted to $H$ is constant. We identify $k[H]$ with $k\left[t, t^{-1}\right]$ and identify the restriction of $y_{i}$ to $H$ with an element $\overline{y_{i}}$ in $k\left[t, t^{-1}\right]$. Our hypotheses imply that the dimension of $k \bar{y}_{i}^{H} \leq N_{i}$ for $i=1, \ldots, n$ 
and Lemma 3, together with property $\left(\mathrm{B}^{\prime}\right)$, then imply that $\overline{y_{i}}$ lies in $k$ for $i$ in $S$.

We now show that property (A) implies property (B). To do this, let $I$ be a set of polynomials that do not have property (B) for $\left(N_{1}, \ldots, N_{n}\right)$ with respect to $S$. We shall show that for the group $G=G_{m}$, there exist elements $u_{i}$ in $k\left[G_{m}\right]$ that satisfy the hypotheses of property (A) but violate the conclusion. Since we are assuming that $I$ does not have property (B), there exist $u_{i}$ in $k\left[t, t^{-1}\right], i=1, \ldots, n$, such that each $u_{i}$ has at most $N_{i}$ nonzero terms and $P\left(u_{1}, \ldots, u_{n}\right)=0$ for all $P$ in $I$, but for some $j$ in $S, u_{j}$ is not a monomial. Identifying $k\left[t, t^{-1}\right]$ with $k\left[G_{m}\right]$, Lemma 3 implies that the dimension of $k u_{i}^{G_{m}} \leq N_{i}$ for $i=1, \ldots, n$. These $u_{i}$ violate the conclusion of property $(A)$. The proof that property $\left(A^{\prime}\right)$ implies property $\left(B^{\prime}\right)$ is similar and is therefore omitted.

3. Differential algebra. Let $k$ be a differential field with commuting derivations $\Delta=\left\{\delta_{1}, \ldots, \delta_{r}\right\}$. The set of elements $c$ in $k$ such that $\delta c=0$ for all $\delta \in \Delta$ is called the constants of $k$. Let $k\left\{Y_{1}, \ldots, Y_{n}\right\}$ be the ring of differential polynomials in the differential indeterminants $Y_{1}, \ldots, Y_{n}$ and let $k\left\{Y_{1}, \ldots, Y_{n}\right\}_{1}$ be the set of all homogeneous linear elements of $k\left\{Y_{1}, \ldots, Y_{n}\right\}$. A differential ideal $\mathfrak{p}$ in $k\left\{Y_{1}, \ldots, Y_{n}\right\}$ is said to be linear [6, p. 150] if it is generated by elements in $k\left\{Y_{1}, \ldots, Y_{n}\right\}_{1}$. If $\mathfrak{p}$ is a linear differential ideal, the codimension of $\mathfrak{p} \cap k\left\{Y_{1}, \ldots, Y_{n}\right\}_{1}$ in $k\left\{Y_{1}, \ldots, Y_{n}\right\}_{1}$ is called the linear dimension of $\mathfrak{p}$ (this need not be finite). Note that if $k$ is an ordinary differential field, a differential ideal $\mathfrak{p}$ of $k\{Y\}$ is linear and of finite linear dimension $l$ if and only if there exists a homogeneous linear differential polynomial $L(y)=y^{(l)}+a_{1} y^{(l-1)}+\cdots+a_{l} y$ in $k\{Y\}$ of order $l$ such that $\mathfrak{p}$ is the differential ideal generated by $L$ [6, p. 155]. We shall state and prove our results for general differential fields and linear differential ideals, but the reader who is only interested in ordinary differential fields and homogeneous linear differential equations, may use this last remark to replace hypotheses such as " $u$ is the zero of a linear differential ideal of linear dimension $l$ " with the more familiar " $u$ satisfies a homogeneous linear differential equation of order $l$ ".

The results of this section depend on the galois theory of linear differential equations, which we shall now review. Let $k$ be as above and assume that the constants $C$ of $k$ are algebraically closed. Let $\mathfrak{p}$ be a linear differential ideal in $k\left\{Y_{1}, \ldots, Y_{n}\right\}$ of finite linear dimension $l$. For any differential extension field $F$ of $k$, the set of zeros of $\mathfrak{p}$ in $F^{n}$ forms a vector space (over the constants of $F$ ) of dimension at most $l[6$, p. 151]. There exists a differential extension field $K$ of $k$, having the same field of constants as $k$, such that the space of zeros of $\mathfrak{p}$ in $K^{n}$ has dimension $l$ and such that $K$ is generated over $k$ by these solutions [6, p. 142]. Furthermore, this extension is unique up to isomorphism. $K$ is referred to as the Picard-Vessiot extension associated with $\mathfrak{p}$. If $\bar{K}$ is a differential extension of $k$, having the same constants as $k$, that is generated over $k$ by zeros of $\mathfrak{p}$, then we can embed $\bar{K}$ in $K$ over $k$. The group of differential automorphisms of $K$ over $k$ acts on the space of zeros of $\mathfrak{p}$ and so can be identified with a group $G$ of invertible matrices in $\mathrm{GL}_{N}(C)$ for some integer $N$. It is known that this group will be closed in the Zariski topology [6, p. 394], and so $G$ is a linear algebraic group defined over $C$ whose $C$-points correspond to differential automorphisms of $K$ over $k$.

There is a galois correspondence between closed subgroups of $G$ and differential fields $F$ with $k \subset F \subset K$. In particular, for $y \in K, \sigma(y)=y$ for all $\sigma \in G$ if and only if $y \in k$. When $k$ is algebraically closed, $G$ is connected [6, p. 402], and, using 
differential galois cohomology, one can show that we may identify $K$ with $k(G)[6$, p. 426]. Since this identification is the key to our method, as it allows us to apply the results of $\S 2$, we shall present an elementary proof.

LEMMA 4. Let $k$ be an algebraically closed differential field of characteristic 0 and $K$ a Picard-Vessiot extension of $k$ with galois group $G$. Then:

(i) There exists an isomorphism $\phi$ of $K$ onto $k(G)$ such that if $\sigma$ is a $C$-point of $G$ then for all $y$ in $K, \phi(\sigma(y))=\rho_{\sigma}^{*}(\phi(y))$.

(ii) For $y$ in $K, y$ is the zero of a linear differential ideal in $k\{Y\}$ of finite linear dimension if and only if $\phi(y) \in k[G]$.

PROOF. For simplicity, we prove this only for ordinary differential fields. The proof in general replaces the use of Wronskians below with determinants of matrices of the form mentioned in Theorem 1 on p. 85 of [6]. We shall first show that for $y \in K, y$ is the zero of a homogeneous linear differential equation if and only if $C y^{G_{C}}$ (the $C$-span of the orbit of $y$ under the action of the $C$-points of $G$ ) has finite dimension. If $y$ satisfies a homogeneous linear differential equation $L(y)=0$, then $y$ belongs to a $G$-stable finite dimensional vector space, namely the space of zeros of $L(y)=0$. Conversely, if $C y^{G_{C}}$ has finite dimension, let $y=y_{1}, \ldots, y_{l}$ be a basis and let

$$
L(Y)=\operatorname{Wr}\left(Y, y_{1}, \ldots, y_{n}\right) / \operatorname{Wr}\left(y_{1}, \ldots, y_{n}\right),
$$

where $\mathrm{Wr}$ is the usual Wronskian determinant. One easily sees that the coefficients of $L(y)$ are left fixed by all elements of the galois group and so lie in $k$. Clearly $L(y)=0$.

From this we see that the set of elements satisfying homogeneous linear differential equations over $k$ forms a differential ring. Assume $K$ is generated (as a differential field) by $y_{1}, \ldots, y_{m}$, a fundamental set of solutions of some homogeneous linear differential equation of order $m$. We may then write

$$
K=k\left(y_{1}, \ldots, y_{m}, y_{1}^{\prime}, \ldots, y_{m}^{\prime}, \ldots, y_{1}^{(m-1)}, \ldots, y_{m}^{(m-1)}\right) \text {. }
$$

Let $S=\left\{y \in K \mid y^{\prime} / y \in k\right\}$. Note that the elements of $S$ satisfy homogeneous linear differential equations over $k$. Since $K$ is finitely generated over $k$, we have by Theorem 1 of $[\mathbf{1 1}]$ that $S$ is a finitely generated abelian group. Let $R=$ $k\left[y_{1}, \ldots, y_{m}, y_{1}^{(m-1)}, \ldots, y_{m}^{(m-1)}, S\right]$. Since $S$ is finitely generated, $R$ is a finitely generated $k$-algebra. Let $V$ be the affine variety whose coordinate ring is $R$. The action of $G$ on $R$ induces an action of $G$ on $V$. We wish to show that $G$ acts transitively and freely on $V$. First of all, for $v \in V$, we claim that the orbit of $v$ is dense in $V$. If not, there is a nonzero $z \in R$ such that $z$ vanishes on the orbit of $v$. Therefore all the elements of $C z^{G_{c}}$ vanish at $v$. Since $z \in R, C z^{G_{c}}$ is finite dimensional, so let $z_{1}, \ldots, z_{r}$ be a basis of $C z^{G_{c}}$ and $I$ be the ideal of $R$ generated by $z_{1}, \ldots, z_{r} . I \neq R$ since $I$ has a zero in $V$. On the other hand, letting $w=\operatorname{Wr}\left(z_{1}, \ldots, z_{r}\right)$, we see that $w$ satisfies $w^{\prime} / w \in k$ (look at the action of the galois group) and $w \in I$ (expand $w$ by minors). Since $1 / w$ is also in $R$, we get $1 \in I$, a contradiction. Therefore the orbit of any element $v \in V$ is dense in $V$. Since the orbits of minimal dimension are closed [5, p. 60], we get that the orbit of any element is all of $V$. This proves that $G$ acts transitively on $V$. To see that $G$ acts freely, let $v \in V$ and assume $v g=v$ for some $g \in G$. Let

$$
v=\left(v_{1}^{(0)}, \ldots, v_{m}^{(0)}, v_{1}^{(1)}, \ldots, v_{m}^{(1)}, \ldots, v_{1}^{(m-1)}, \ldots, v_{m}^{(m-1)}, \ldots\right)
$$


If we write the first $m^{2}$ entries as a matrix $\left(v_{i}^{(j)}\right), 1 \leq i \leq m, 0 \leq j \leq m-1$, then $g$ acts as an $m \times m$ matrix by multiplication on the right of the matrix. Since $v g=v$, we have $\left(v_{i}^{(j)}\right) g=\left(v_{i}^{(j)}\right)$. Since $1 / \operatorname{Wr}\left(y_{1}, \ldots, y_{m}\right) \in R, \operatorname{det}\left(v_{i}^{(j)}\right) \neq 0$, so $g=$ id. Therefore, $G$ acts freely. Let $v \in V$ be a $k$-point of $V$. The map from $G$ to $V$ given by $g \rightarrow v g$ induces an isomorphism $\phi$ of $k[V]$ onto $k[G]$. This extends to the quotient fields and clearly has the properties claimed in (1).

To see (ii), we have already noted that, for $u \in k(G), u \in k[G]$ if and only if $k u^{G}$ hs finite dimension. Since the $C$-points of $G$ are dense in $G, k u^{G}$ has finite dimension if and only if $C u^{G_{c}}$ has finite dimension. We have shown above that for $y \in K, y$ satisfies a homogeneous linear differential equation if and only if $C u^{G_{c}}$ has finite dimension. (ii) now follows from (i).

For the remainder of this section, except where otherwise noted, let $k$ be an algebraically closed differential field of characteristic 0 with derivations $\Delta=\left\{\delta_{1}, \ldots, \delta_{r}\right\}$. Let $N_{1}, \ldots, N_{n}$ be positive integers, $S$ a subset of $\{1, \ldots, n\}$ and $I$ a set of polynomials in $k\left[Y_{1}, \ldots, Y_{n}\right]$. We say that $I$ satisfies property $(\mathrm{C})$ (resp. $\left(\mathrm{C}^{\prime}\right)$ ) for $\left(N_{1}, \ldots, N_{n}\right)$ with respect to $S$ if:

For any differential extension $K$ of $k$ having the same field of constants as $k, y_{1}, \ldots, y_{n} \in K-\{0\}$, linear differential ideals $\mathfrak{p}_{1}, \ldots, \mathfrak{p}_{n}$ in $k\{Y\}$ such that $y_{i}$ is a zero of $\mathfrak{p}_{i}$ and the linear differential dimension of $\mathfrak{p}_{i}$ is $\leq N_{i}$ for $i=1, \ldots, n$, if $P\left(y_{1}, \ldots, y_{n}\right)=0$ for all $P$ in $I$, then $\delta y_{i} / y_{i}$ is in $k$ for $i$ in $S$ and $\delta$ in $\Delta$ (resp. $y_{i}$ is in $k$ for all $i$ in $S$ ).

THEOREM 2. Let $k,\left(N_{1}, \ldots, N_{n}\right), S$ and $I$ be as above.

(a) If I has property (B) (resp. $\left.\left(\mathrm{B}^{\prime}\right)\right)$ for $\left(N_{1}, \ldots, N_{n}\right)$ with respect to $S$, then $I$ has property $(\mathrm{C})$ (resp. $\left.\left(\mathrm{C}^{\prime}\right)\right)$ for $\left(N_{1}, \ldots, N_{n}\right)$ with respect to $S$.

(b) Assume $k$ is an algebraically closed ordinary differential field with derivation $\delta$ and assume there exists $u \in k$ such that $\delta Y-u Y=0$ has no nonzero solution in $k$. If I has property $(\mathrm{C})$ (resp. $\left.\left(\mathrm{C}^{\prime}\right)\right)$ for $\left(N_{1}, \ldots, N_{n}\right)$ with respect to $S$, then $I$ has property (B) (resp. $\left.\left(\mathrm{B}^{\prime}\right)\right)$ for $\left(N_{1}, \ldots, N_{n}\right)$ with respect to $S$.

PROOF. (a) We shall show that if $I$ has property (A) (resp. $\left.\left(\mathrm{A}^{\prime}\right)\right)$ for $\left(N_{1}, \ldots, N_{n}\right)$ with respect to $S$ then $I$ has property $(\mathrm{C})$ (resp. $\left.\left(\mathrm{C}^{\prime}\right)\right)$ for $\left(N_{1}, \ldots, N_{n}\right)$ with respect to $S$. Theorem 1 then allows us to conclude (a) above. Let $K$ be a differential extension of $k$ with the same field of constants $C$ as $k$. Let $y_{1}, \ldots, y_{r} \in K-\{0\}$ and let $\mathfrak{p}_{1}, \ldots, \mathfrak{p}_{n}$ be linear differential ideals in $k[Y]$ such that $y_{i}$ is a zero of $\mathfrak{p}_{i}$, such that the linear differential dimension of $\mathfrak{p}_{i} \leq N_{i}$ and such that $P\left(y_{1}, \ldots, y_{n}\right)=0$ for all $P$ in $I$. Since $K$ has the same field of constants as $k$, we may assume that $y_{1}, \ldots, y_{n}$ lie in a Picard-Vessiot extension $F$ of $k$. Let $G$ be the galois group of $F$ over $k$. Since $k$ is algebraically closed, $G$ is a connected $C$-group and $F$ may be identified with $k(G)$. Furthermore, for any $g$, a $C$-point of $G$, the galois action of $g$ on $F$ is given by $\rho_{g}^{*}$. Since the linear dimension of each $\mathfrak{p}_{i} \leq N_{i}$, we have that the dimension of $C y_{i}^{G_{C}} \leq N_{i}$, where $G_{C}$ is the group of $C$-points of $G$. Since $G_{C}$ is dense in $G$, we have that the dimension of $k y_{i}^{G} \leq N_{i}$. If $I$ has property $\left(\mathrm{A}^{\prime}\right)$ for $\left(N_{1}, \ldots, N_{n}\right)$ with respect to $S$, then for each $i$ in $S, y_{i}$ is in $k$, so $I$ has property $\left(\mathrm{C}^{\prime}\right)$ for $\left(N_{1}, \ldots, N_{n}\right)$ with respect to $S$. If $I$ has property (B) for $\left(N_{1}, \ldots, N_{n}\right)$ with respect to $S$, then we can conclude that for each $i$ in $S, y_{i}$ is a $k$-multiple of 
a character of $G$. Fix some $i$ in $S$ and denote $y_{i}$ by $y$ and $\mathfrak{p}_{i}$ by $\mathfrak{p}$. For any $g \in G$, $\rho_{g}^{*}(Y)=a_{g} y$ for some $a_{g}$ in $k$. We claim that for $g \in G_{C}, a_{g} \in C$. Fix some $\delta$ in $\Delta$. Since $\mathfrak{p}$ has finite linear dimension, there exist $a_{l-1}, \ldots, a_{0}$ in $k$ such that $L(y)=\delta^{(l)} y+a_{l-1} \delta^{(l-1)} y+\cdots+a_{0} y=0$. Among all such equations, choose one with $l$ minimal. If $g \in G_{C}$, then $L\left(\rho_{g}^{*} y\right)=0$ so

$$
\begin{aligned}
0 & =\delta^{(l)}\left(a_{g} y\right)+a_{n-1} \delta^{(l)}\left(a_{g} y\right)+\cdots+a_{0}\left(a_{g} y\right) \\
& =a_{g} \delta^{(l)} y+\left(n \delta a_{g}+a_{n-1} a_{g}\right) \delta^{(l-1)} y+\cdots
\end{aligned}
$$

By minimality we have $a_{g} a_{n-1}=n \delta a_{g}+a_{n-1} a_{g}$ or $\delta a_{g}=0$. In particular, this implies that $\delta y / y$ is fixed by all elements of $G_{C}$ and so must lie in $k$. Therefore $I$ has property (A) for $\left(N_{1}, \ldots, N_{n}\right)$ with respect to $S$.

(b) We shall show that if $I$ does not have property (B) for $\left(N_{1}, \ldots, N_{n}\right)$ with respect to $S$, then $I$ does not have property (C) for $\left(N_{1}, \ldots, N_{n}\right)$ with respect to $S$. Let $u_{1}, \ldots, u_{n}$ be nonzero elements of $k\left[t, t^{-1}\right]$ such that each $u_{i}$ has $\leq N_{i}$ nonzero terms and $P\left(u_{1}, \ldots, u_{n}\right)=0$ for all $P$ in $I$. Assume that for some $i$ in $S, u_{i}$ is not a monomial. We extend $\delta$ to $K=k(t)(t$ transcendental over $k)$ by letting $\delta t=u t$. Since $\delta Y-u Y$ has no solutions in $k$ and $k$ is algebraically closed, $k$ and $K$ have the same field of constants $[9$, p. 172]. One can easily check that an element of the form $y=\sum a_{j} t^{j}$ having at most $N_{i}$ nonzero terms satisfies a homogeneous linear differential equation of order at most $N_{i}$. Furthermore, if $\delta y / y=a \in k$, then $y$ must be a monomial. To see this, observe that $0=\delta y-a y=\sum\left(\delta a_{j}-a_{j}(a-j u)\right) t^{j}$. If $a_{i}$ and $a_{j}$ are nonzero for $i \neq j$, then $\delta v / v=u$, where $v=\left(a_{i} a_{j}^{-1}\right)^{1 /(j-i)}$, contradicting our assumption that $\delta Y-u Y=0$ has no solutions in $k$.

The proof that Property $\left(\mathrm{C}^{\prime}\right)$ implies property $\left(\mathrm{B}^{\prime}\right)$ is similar and is therefore omitted.

PROPOSITION 3 (BIS). Let $k \subset K$ be differential fields of characteristic 0 with the same field of constants and $k$ algebraically closed. Let $\mathfrak{p}$ be a linear differential ideal of finite linear dimension and let $\left(y_{1}, y_{2}, y_{3}\right) \in K^{3}$ be a zero of $\mathfrak{p}$. Assume that $y_{1}=y_{2} y_{3}^{m}$ for some positive integer $m$. If the linear dimension of $\mathfrak{p} \cap k\left\{Y_{1}\right\} \leq m$, then $\delta y_{3} / y_{3}$ is in $k$ for all $\delta \in \Delta$.

Proof. For each $i, i=1,2,3, \mathfrak{p} \cap k\left\{Y_{i}\right\}=\mathfrak{p}_{i}$ is a linear differential ideal of finite linear dimension. For arbitrary positive integers $N_{2}$ and $N_{3}$, we have seen that $I=\left\{Y_{1}-Y_{2} Y_{3}^{m}\right\}$ has property (B) for $\left(m, N_{2}, N_{3}\right)$ with respect to $\{3\}$. Theorem 2 gives us the desired conclusion

Proposition 3 in the introduction is just the ordinary differential versions of this last result. One can also deduce from this partial differential versions of Propositions 1 and 2. For example, assume that $k \subset K$ are differential fields of characteristic zero with the constants of $K$ algebraic over the constants of $k$. If $\left(y_{1}, y_{2}\right) \in K^{2}$ is a zero of a linear differential ideal of finite linear dimension and $y_{1} y_{2}=1$, then $\delta y_{i} / y_{i}$ is algebraic over $k$ for all $\delta$ in $\Delta$.

Proposition 4. Let $k \subset K$ be differential fields of characteristic 0 with the same field of constants and $k$ algebraically closed. Let $I$ be an ideal in $k\left[Y_{1}, \ldots, Y_{n}\right]$ whose set of zeros in $k^{n}$ is a curve of genus $\geq 1$. If $\mathfrak{p} \subset k\left\{Y_{1}, \ldots, Y_{n}\right\}$ is a linear differential ideal of finite linear dimension and $\left(y_{1}, \ldots, y_{n}\right) \in K^{n}$ is a zero of both $I$ and $\mathfrak{p}$, then for each $i=1, \ldots, n, y_{i}$ is in $k$. 
Proof. Since $I$ defines a curve of genus $\geq 1$, if $u_{1}, \ldots, u_{n} \in k(t)$ and

$$
P\left(u_{1}, \ldots, u_{n}\right)=0 \text { for all } P \text { in } I
$$

then $u_{1}, \ldots, u_{n}$ are all in $k$. Therefore, $I$ has property $\left(\mathrm{B}^{\prime}\right)$ for all $\left(N_{1}, \ldots, N_{n}\right)$ with respect to $\{1, \ldots, n\}$. Since $\mathfrak{p}$ has finite linear dimension, so does $\mathfrak{p}_{1}=\mathfrak{p} \cap k\left\{Y_{i}\right\}$ for $i=1, \ldots, n$. Therefore, Theorem 2 implies each $y_{i}$ is in $k$.

For example, consider the rational functions $\mathbf{C}(x)$ with derivation $d / d x$. If $f(x)$ is an algebraic function of genus $\geq 1$ (e.g., $f(x)=\left(1-x^{n}\right)^{1 / n}$ with $n \geq 3$ ) and $y$ and $f(y)$ satisfy linear differential equations over $\mathbf{C}(x)$, then Proposition 4 implies that $y(x)$ is an algebraic function.

Our techniques also allow us to deal with certain algebraic relations that involve derivatives. If $\Delta$ is a set of derivations, let $\theta$ be the free commutative multiplicative semigroup generated by the elements of $\Delta$. If $k \subset K$ are differential fields and $y \in K$, we say $y$ is monic over $k$ if $y^{m}-f\left(\theta_{1} y, \ldots, \theta_{s} y\right)=0$, where $\theta_{i} \in \Theta$ for $i=1, \ldots, s$ and $f$ a polynomial of total degree strictly less than $m$.

Proposition 5. Let $k \subset K$ be differential fields of characteristic 0 having the same field of constants, with $k$ algebraically closed. If $k \in K$ is a zero of a linear differential ideal in $k\{Y\}$ of finite linear dimension and $y$ is monic over $k$, then $y \in k$.

Proof. We may assume $K$ is a Picard-Vessiot extension of $k$ with galois group $G$. $G$ is connected, and we may identify $K$ with $k(G)$ and $y$ with an element of $k[G]$. Let $C$ be the field of constants of $k$. We must show that for any $g \in G_{C}, \rho_{g}^{*}(y)=y$. First assume that $g$ is unipotent. $g$ belongs to a closed subgroup $H$ of $G$ isomorphic to $G_{a}$. Let $F$ be the fixed field of $H$. We shall show that $y$ is in $F$. Corollary 2 of $[6$, p. 427] implies that $K=F(t)$ with $\delta t \in F$ for all $\delta$ in $\Delta$. Furthermore, $y \in F[t]$. For $u \in F[t]$, let $o(u)$ denote the degree of $u$ in $t$. For $\theta \in \Theta$ we have $o(\theta(u)) \leq o(u)$. If $o(y)>0$, then $o\left(f\left(\theta_{1}(y), \ldots, \theta_{s}(y)\right)\right)<m(o(y))=o\left(y^{m}\right)$. Therefore $o(y)=0$; i.e., $y \in F$. Now assume $g$ is semisimple. Since $g$ lies in a torus, to show that $\rho_{g}^{*}(y)=y$, it is enough to show that for any subgroup $H$ of $G$ isomorphic to $G_{m}$, $H$ lies in the fixed field $F$ of $H$. Again by Corollary 2 of $[6$, p. 427], $K=F(t)$ with $\delta t / t$ in $F$ for all $\delta$ in $\Delta$. Furthermore, $y \in F\left[t, t^{-1}\right]$. For $u \in F\left[t, t^{-1}\right]$, we let $o_{1}(u)$ be the highest power of $t$ occurring in $u$ and $o_{2}(u)$ be the highest power of $t^{-1}$ appearing in $u$. For $\theta \in \Theta$ we have $o_{1}(\theta(u)) \leq o_{1}(u)$ and $o_{2}(\theta(u)) \leq o_{2}(u)$. Arguing as before, we see that $o_{1}(y)=o_{2}(y)=0$, so $y \in F$. Since any element of $G$ is the product of unipotent and semisimple elements, we see that $y$ is left fixed by all of $G$. Therefore $y \in k$.

When $k$ is an ordinary differential field, this result (in greater generality and without the assumption on constants) was proved by S. Morrison [2, 8]. If $u$ satisfies a linear differential equation, then $y=u^{\prime} / u$ satisfies a Riccati equation, so $y$ is monic. Therefore if $u$ belongs to a differential extension of $k$ having the same constants as $k$ and $u$ and $u^{\prime} / u$ satisfy linear differential equations over $k$, then $u$ is algebraic over $k$. More concretely, if $y(x)$ and $e^{\int y(x)}$ satisfy linear differential equations over $\mathbf{C}(x)$, then $y$ is an algebraic function. 


\section{REFERENCES}

1. A. Bialynicki-Birula, On galois theory of fields with operators, Amer. J. Math. 84 (1962), 89-109.

2. P. Blum, Rational functions on differentially closed sets, Amer. J. Math. 94 (1972), 676687.

3. W. A. Harris, Jr. and Y. Sibuya, The reciprocals of solutions of linear ordinary differential equations, Adv. in Math. 58 (1985), 119-132.

4.

5. J. E. Humphreys, Linear algebraic groups, Springer-Verlag, New York, 1975.

6. E. R. Kolchin, Differential algebra and algebraic groups, Academic Press, New York, 1973.

7. A. Magid, Finite generation of class groups of rings of invariants, Proc. Amer. Math. Soc. 60 (1976), 45-48.

8. S. D. Morrison, Extension of differential places, Amer. J. Math. 100 (1978), 245-261.

9. R. H. Risch, The problem of integration in finite terms, Trans. Amer. Math. Soc. 139 (1969), 167-189.

10. M. Rosenlicht, Toroidal algebraic groups, Proc. Amer. Math. Soc. 12 (1961), 984-988.

11. __ Differential extension fields of exponential type, Pacific J. Math. 57 (1975), 289300.

12. S. Sperber, On solutions of differential equations which satisfy certain algebraic relations, preprint.

Department of Mathematics, BoX 8205, North Carolina State University, RALEIGH, NORTH CAROLINA 27695-8205 